\title{
QUALIDADE DO SÊMEN DE TAMBAQUI (Colossoma macropomum) CRIOPRESERVADO EM DIFERENTES CONCENTRAÇÕES DE GEMA DE OVO
}

\author{
QUALITY OF TAMBAQUI (Colossoma macropomum) SEMEN \\ CRYOPRESERVED IN DIFFERENT CONCENTRATIONS OF EGG YOLK \\ João Paulo Silva Pinheiro ${ }^{1}$ \\ Liliane Veras Leite-Castro ${ }^{1}$ \\ Fátima de Cássia Evangelista de Oliveira ${ }^{1}$ \\ Francisco Renan Aragão Linhares ${ }^{1}$ \\ Júlia Trugílio Lopes ${ }^{1}$ \\ Carminda Sandra Brito Salmito-Vanderley ${ }^{1 *}$ \\ ${ }^{1}$ Universidade Estadual do Ceará, Fortaleza, CE, Brasil. \\ *Autora para correspondência - ssalmito@yahoo.com
}

\section{Resumo}

O objetivo deste trabalho foi avaliar o efeito da adição de gema de ovo (GO) sobre a cinética dos espermatozoides de tambaquis após a criopreservação. Utilizaram-se vinte machos de tambaquis $(\mathrm{n}=$ 4 pools), que foram induzidos hormonalmente com extrato hipofisário de carpa, para espermiação. Quatorze horas após a indução, realizou-se a coleta seminal. O sêmen de cada pool foi diluído em Ringer adicionado de 10\% de dimetilsulfóxido (DMSO) acrescido ou não de GO (T1: sem acréscimo de GO; T2: com 5\% de GO e T3: com 10\% de GO). O sêmen tratado foi envasado em palhetas de 0,5 mL, congelado em vapor de nitrogênio líquido (dry shipper-30 $\mathrm{min} /-153{ }^{\circ} \mathrm{C}$ ) e posteriormente transferidas para nitrogênio líquido. As palhetas foram descongeladas em banho-maria a $37^{\circ} \mathrm{C} / 30$ segundos. A taxa de motilidade $(\%)$ e a velocidade curvilinear espermática $(\mu \mathrm{m} / \mathrm{s})$ foram analisadas em sistema computadorizado (CASA). Os dados foram expressos em média \pm desvio padrão e foi aplicado o teste de Tukey $(\mathrm{P}<0,05)$. Houve uma redução significativa na porcentagem de espermatozoides móveis e velocidade curvilinear após a adição de GO independente da concentração. Logo, a adição de GO ao Ringer + DMSO teve efeito negativo sobre a motilidade do sêmen congelado de tambaqui.

Palavras-chave: congelação; crioprotetor extracelular; reprodução.

\begin{abstract}
The aim of this study was to evaluate the effect of egg yolk (EY) addition on the sperm kinects after cryopreservation. We used twenty tambaqui males ( $\mathrm{n}=4$ pools), which were induced homonally to spermiation with carp pituitary extract. Fourteen hours after induction, a seminal collection was performed. The semen from each pool was diluted with Ringer added 10\% DMSO on the presence or absence of egg yolk (T1: no additional EY; T2: 5\% EY; and T3: 10\% EY). The treated semen was loaded in $0.5 \mathrm{~mL}$ straws, frozen in a nitrogen vapor vessel (dry shipper) $\left(30 \mathrm{~min} /-153^{\circ} \mathrm{C}\right)$, and then transferred to liquid nitrogen. Straws were thawed in a water bath at $37^{\circ} \mathrm{C} / 30$ seconds. The analyses of motility rate $(\%)$ and sperm curvilinear velocity $(\mu \mathrm{m} / \mathrm{s})$ were performed on computerized system (CASA). Data were expressed as mean \pm standard deviation, and Tukey test was applied $(\mathrm{P}<0.05)$. There was a significant reduction in the percentage of motile spermatozoa and curvilinear velocity after the addition of egg yolk to the crioprotection solution, regardless of the concentration. Therefore, the addition of EY to Ringer + DMSO had negative effect on motility of tambaqui frozen sperm.
\end{abstract}

Keywords: extracellular cryoprotectant; freezing; reproduction. 


\section{Introdução}

O tambaqui (Colossoma macropomum) é um peixe Characiforme endêmico da região amazônica do Brasil. Também é encontrado em outras regiões como o Nordeste, em virtude de sua rusticidade para criação em cativeiro e do grande apelo culinário ${ }^{(1,2)}$, sendo, assim, uma das espécies mais cultivadas no Brasil ${ }^{(3)}$.

Em virtude da grande demanda de mercado, o tambaqui foi eleito como uma das espécies prioritárias para cultivo ${ }^{(3)}$; entretanto, com a pesca predatória e com a construção de barragens em rios, que impedem sua reprodução no ambiente natural, os estoques de tambaquis estão sendo reduzidos assim como sua capacidade de reposição ${ }^{(4,5)}$. Logo, o uso da técnica de criopreservação de sêmen poderá facilitar a formação de famílias para melhoramento e conservação da diversidade genética da espécie, permitindo a constituição de bancos de germoplasma ${ }^{(6,7)}$.

O sucesso da criopreservação está relacionado a diversos fatores, entre eles a composição do diluente e o crioprotetor intracelular e/ou extracelular utilizado. Portanto, busca-se a associação ideal entre esses elementos com o intuito de garantir uma boa qualidade seminal pós-descongelação ${ }^{(8-10)}$.

Diluentes mais complexos com diferentes concentrações de íons podem fornecer maior nutrição e proteção aos espermatozoides, como o Ringer modificado para peixes, composto por $\mathrm{NaCl}, \mathrm{KCl}$, $\mathrm{NaHCO}_{3} \mathrm{e} \mathrm{CaCl}_{2}$, já testado para outras espécies de água doce, apresentando boas taxas de motilidade espermática ${ }^{(11-13)}$.

De acordo com Salmito-Vanderley et al. ${ }^{(8)}$, um dos crioprotetores intracelulares mais utilizados com sucesso na congelação seminal de peixes é o dimetilsulfóxido (DMSO), já testado para várias espécies de Characiformes ${ }^{(14-17)}$.

Quanto ao crioprotetor extracelular, a gema de ovo de galinha é bastante utilizada, pois possui em sua composição a fosfatidilcolina (lecitina) e lipoproteínas, que atuam na estabilização da membrana reduzindo as crioinjúrias ${ }^{(5)}$. De fato, em muitos trabalhos de criopreservação de sêmen de Characídeos, a gema de ovo é usada em associação à solução de glicose 5\% (diluente) e ao crioprotetor intracelular DMSO, com resultados controversos entre os autores ${ }^{(6,16,18-21)}$.

Como não há trabalhos com a espécie C. macropomum que demonstrem se a utilização da gema de ovo associada ao diluente Ringer é eficaz quanto à cinética espermática pós-descongelação, o objetivo deste trabalho foi avaliar o efeito da associação do diluidor Ringer + DMSO com diferentes concentrações de gema de ovo sobre a taxa de motilidade e velocidade dos espermatozoides de tambaqui após a criopreservação.

\section{Material e Métodos}

Este trabalho foi aprovado pelo Comitê de Ética para o uso de animais da Universidade Estadual do Ceará (UECE), processo ${ }^{\circ} 09144388-1$.

Para o desenvolvimento do estudo, foram utilizados vinte machos adultos, com idade média de três anos de idade, peso médio de $6,12 \pm 0,41 \mathrm{~kg}$ e comprimento médio de $61,95 \pm 4,52 \mathrm{~cm}$, provenientes do Centro de Pesquisa em Aquicultura (CPAq) do Departamento Nacional de Obras Contra as Secas (DNOCS), localizado no município de Pentecoste, Ceará.

Os peixes foram induzidos hormonalmente à espermiação com uma dose de extrato hipofisário de carpa ( $\left.2 \mathrm{mg} \cdot \mathrm{kg}^{-1}\right)$, intracelomático, na base da nadadeira peitoral, sendo realizada a coleta seminal quatorze horas após essa indução. A fim de reduzir o estresse durante a coleta seminal, cada animal foi sedado com solução à base de óleo de cravo (Eugenol; União Vegetal Suplementos Nutricionais Ltda) na proporção 1:10:10000 (eugenol: álcool absoluto: água) até que apresentasse a perda do equilíbrio (ventre voltado para cima). Após esta etapa, o animal foi imediatamente retirado do tanque, com os olhos vendados com pano úmido, colocado sobre esponja e submetido à coleta de sêmen. Após este procedimento, o mesmo era devolvido ao tanque de manuseio para se recuperar totalmente da 
sedação.

Na coleta seminal, a papila urogenital foi cuidadosamente limpa e enxuta e uma pressão abdominal foi realizada no sentido ântero-posterior para liberação do sêmen. Logo após, foram aferidos o volume $(\mathrm{mL})$ com tubos graduados e o $\mathrm{pH}(0-14)$ mediante uso de fitas reagentes. As amostras seminais foram avaliadas subjetivamente ao microscópio de luz (400X). A primeira análise foi feita com o sêmen puro, sem adição de água do tanque, e a segunda após sua ativação com água do tanque $(2 \mu \mathrm{L}$ de sêmen: $100 \mu \mathrm{L}$ de água). Amostras seminais, cuja motilidade foi nula na primeira análise e que após ativação mostrou motilidade seminal igual ou superior a $90 \%$, foram selecionadas para a formação de quatro pools de sêmen $(\mathrm{n}=4)$, em que cada pool foi composto pelo sêmen de cinco machos diferentes em iguais proporções.

Uma alíquota de $100 \mu \mathrm{L}$ foi destinada para mensuração da osmolaridade (mOsm/Kg) seminal de cada animal (Osmometer Automatic, Roebling, Alemanha). Alíquotas de $1 \mu \mathrm{L}$ foram retiradas e diluídas em $4000 \mu \mathrm{L}$ de solução salina formolizada a 1\% para contagem de espermatozoides em Câmara de Neubauer.

No que se refere ao processo de criopreservação, utilizou-se Ringer modificado para peixes $(\mathrm{NaCl}$, $6,5 \mathrm{~g} / \mathrm{L} ; \mathrm{KCl}, 3,0 \mathrm{~g} / \mathrm{L} ; \mathrm{NaHCO}_{3}, 0,2 \mathrm{~g} / \mathrm{L} ; \mathrm{CaCl}_{2} \cdot 6 \mathrm{H}_{2} \mathrm{O}, 0,3 \mathrm{~g} / \mathrm{L} ; \mathrm{pH} 7,8 ; 300 \mathrm{mOsmol} / \mathrm{Kg}$ ) (RANA e MCANDREW, 1989) como diluente, dimetilsulfóxido a 10\% (DMSO) como crioprotetor intracelular e gema de ovo a $5 \%$ ou $10 \%$ como crioprotetor extracelular. Tais substâncias foram combinadas constituindo os seguintes tratamentos: Ringer + DMSO (T1); Ringer + DMSO + gema 5\% (T2); Ringer + DMSO + gema 10\% (T3). As amostras foram diluídas na proporção de 1:4 (sêmen:diluidor) e envasadas em palhetas plásticas de $0,5 \mathrm{~mL}$.

Posteriormente, as palhetas foram seladas com álcool polivinílico e transferidas para vapor de nitrogênio em dry shipper (Taylor-Wharton, CX100; $-153^{\circ} \mathrm{C}$ ) por 30 minutos e, em seguida, imersas em botijão de nitrogênio líquido $\left(-196^{\circ} \mathrm{C}\right)$. As amostras congeladas foram transportadas para o Laboratório de Biotecnologia da Reprodução de Peixes (LBRP), localizado na Universidade Estadual do Ceará (UECE), Fortaleza, Brasil.

Após quinze dias da criopreservação, as palhetas foram descongeladas em banho-maria a $37{ }^{\circ} \mathrm{C}$ por 30 segundos. Em seguida, o sêmen ativado com $\mathrm{NaCl}(50 \mathrm{mM})$ foi submetido à análise da cinética espermática (motilidade total e velocidade curvilinear dos espermatozoides - VCL) com auxílio da câmara de Makler $^{\circledR}$ acoplada a um microscópio óptico de contraste de fase e a um sistema computadorizado de análise seminal (CASA) (Computer Assisted Sperm Analyzer - 400×magnificância; software $\mathrm{SCA}^{\circledR}$ - Sperm Class Analyzer ${ }^{\circledR}$ ).

Para avaliar possíveis diferenças entre os tratamentos, os dados de motilidade e de velocidade curvilinear foram submetidos à análise de variância e as médias comparadas pelo teste de Tukey, com um nível de significância de 5\%, por meio do Statistical Analysis System (SAS), 2003. Os dados de $\mathrm{pH}$ e osmolaridade foram expressos como média \pm desvio-padrão.

\section{Resultados e Discussão}

O sêmen de tambaqui apresentou coloração esbranquiçada e aspecto leitoso, com volume de 4 a $6 \mathrm{~mL}$ por macho, com concentração espermática entre 12 a $27 \times 10^{9}$ espermatozoides $/ \mathrm{mL}$, osmolaridade de $298,2 \pm 11,7 \mathrm{mOsmol}$ e de $\mathrm{pH} 8,0 \pm 0,5$.

Viveiros e Godinho ${ }^{(22)}$ mencionaram o volume seminal como um fator importante no processo reprodutivo e, tanto em espécies migratórias na época de reprodução quanto em animais induzidos com hormônio, este parâmetro é bastante variável. Esta característica é influenciada por muitos fatores tais como espécie, estado nutricional, peso, idade, tipo e dosagem da indução hormonal, além do método de coleta de sêmen ${ }^{(2)}$.

Farias et al. ${ }^{(23)}$ e Menezes et al. ${ }^{(24)}$ obtiveram, com tambaqui, concentrações espermáticas da ordem de $35 \times 10^{9}$ espermatozoides $/ \mathrm{mL}$, portanto superiores às encontradas neste trabalho, provavelmente, porque estes autores usaram uma dosagem hormonal inferior ( $1 \mathrm{vs} 2 \mathrm{mg} \mathrm{kg}^{-1}$ ). Segundo Viveiros et al. ${ }^{(25)}$, doses hormonais maiores promovem uma maior hidratação testicular, gerando um aumento no volume do fluido seminal e diminuição da concentração espermática. No entanto, as concentrações 
espermáticas obtidas no presente estudo foram similares às encontradas por Vieira et al. ${ }^{(1)}$ e Leite et al. ${ }^{(9)}$, e superiores às encontradas por Maria et al. ${ }^{(2)}$.

$\mathrm{O} \mathrm{pH}$ e a osmolaridade do sêmen apresentaram valores dentro dos padrões para peixes de água doce, segundo Millinger ${ }^{(26)}$. O conhecimento da osmolaridade média do sêmen de peixe teleósteos, como no caso do tambaqui, é de extrema importância na reprodução assistida, uma vez que seus espermatozoides permanecem quiescentes na osmolaridade do plasma seminal ${ }^{(27)}$, fator crítico aos espermatozoides que, após a ativação, tem uma viabilidade média de aproximadamente 2 minutos. Dentre os fatores que ocasionam a ativação da motilidade espermática está o choque osmótico que os espermatozoides sofrem ao encontrarem a água doce (meio hipotônico; $4 \mathrm{mOsm} / \mathrm{Kg}$ ) ou salgada (meio hipertônico, $1.000 \mathrm{mOsm} / \mathrm{Kg}$ ), se teleósteos continentais ou marinhos ${ }^{(28)}$.

Um fator determinante para o sucesso da criopreservação de sêmen é o desenvolvimento de diluidores seminais com $\mathrm{pH}$ e, principalmente, osmolaridade ajustados para a espécie, a fim de manter a quiescência dos espermatozoides antes da etapa de descongelação, de modo a não favorecer ativação espontânea e, com isso, perda de viabilidade espermática.

Os dados de motilidade e velocidade espermática, obtidos pelo sistema de análise seminal computadorizado (CASA) após a descongelação estão descritos na Tabela 1. Foi observado que o tratamento com Ringer + DMSO sem acréscimo de gema (T1) apresentou uma média de 20,4\% de espermatozoides móveis após a descongelação, sendo este valor superior aos encontrados para os tratamentos com adição de gema $(\mathrm{P}<0,05)$.

Tabela 1: Motilidade e velocidade curvilinear (VCL) espermática após a descongelação do sêmen de tambaqui em diferentes concentrações de gema de ovo

\begin{tabular}{llcll}
\hline Diluente & $\begin{array}{l}\text { Crioprotetor } \\
\text { intracelular }\end{array}$ & Concentração de gema de ovo & Motilidade (\%) & VCL $(\boldsymbol{\mu m} / \mathbf{s})$ \\
\hline Ringer & DMSO & $0 \%$ & $20,4 \pm 2,9^{\mathrm{a}}$ & $32,1 \pm 2,7^{\mathrm{a}}$ \\
& & $5 \%$ & $15,8 \pm 2,7^{\mathrm{b}}$ & $23,6 \pm 1,3^{\mathrm{b}}$ \\
& & $10 \%$ & $13,9 \pm 2,9^{\mathrm{b}}$ & $22,9 \pm 1,5^{\mathrm{b}}$ \\
\hline
\end{tabular}

Letras diferentes indicam diferença estatística entre as linhas $(\mathrm{P}<0,05)$ comparadas pelo Teste de Tukey.

A adição de gema de ovo promoveu uma diminuição significativa na motilidade espermática, independente da concentração utilizada ( 5 ou 10\%), apresentando em média $15,8 \%$ de espermatozoides móveis no T2 (com 5\% de gema), e 13,9\% no T3 (com 10\% de gema) ( $\mathrm{P}>0,05)$. A redução na qualidade seminal pós-descongelação também foi observada em alguns trabalhos, que apresentaram baixas taxas de motilidade espermática ${ }^{(6,9,15,29)}$ e significativo aumento nas alterações morfológicas ${ }^{(30)}$ ao se utilizar a gema de ovo associada a diferentes crioprotetores, inclusive DMSO.

Em estudos sobre criopreservação de sêmen de tambaqui, Menezes et al. ${ }^{(24)}$ observaram motilidade entre 5 à 10\% de espermatozoides após a descongelação do sêmen diluído apenas com DMSO. No presente estudo, foi encontrada uma taxa de espermatozoides móveis superior $(20 \%)$ a esse estudo utilizando apenas o $\mathrm{DMSO}^{(24)}$; no entanto, foi inferior ao encontrado por Carneiro et al. ${ }^{(6)}$ ao utilizarem Glicose com DMSO e $10 \%$ gema de ovo (36\%). Essa variação pode ter ocorrido em virtude das diferentes condições de manejo dos reprodutores ${ }^{(9)}$ e pela utilização de diferentes taxas de diluição do sêmen ${ }^{(31)}$.

Além da composição do meio diluidor, um dos fatores que influenciam a qualidade do sêmen criopreservado é a motilidade espermática inicial, ${ }^{(8,32)}$ cuja análise ficou mais precisa ${ }^{(33)}$ com o advento de métodos computadorizados de avaliação da motilidade. Outros aspectos do movimento espermático passaram a ser relacionados com a capacidade fertilizante do sêmen ${ }^{(9)}$. Dentre esses aspectos, um dos mais analisados é a velocidade espermática, principalmente a velocidade curvilinear (VCL) que no sêmen de Prochilodus lineatus após a descongelação apresentou altos níveis de correlação com a taxa de fertilização ${ }^{(34)}$. No presente estudo, a VCL do tratamento com ausência de gema foi em média 34,1 $\mu \mathrm{m} / \mathrm{s}$, sendo superior às encontradas para os tratamentos com acréscimo de 5 e $10 \%$ de gema $(26,6$ 
$\mu \mathrm{m} / \mathrm{s}$ e $23,1 \mu \mathrm{m} / \mathrm{s}$, respectivamente $)(\mathrm{P}<0,05)$.

NASCIMENTO et al. ${ }^{(35)}$ utilizaram o CASA para avaliação da motilidade e velocidade espermática de pirapitinga (Piaractus brachypomus) após a descongelação e também observaram variação da VCL entre os diferentes tratamentos. Essa análise da VCL é de suma importância, já que é a velocidade da trajetória real do espermatozoide ${ }^{(36)}$, e está relacionada com a capacidade fertilizante do sêmen de peixes $^{(34)}$.

\section{Conclusão}

Nas condições em que se deu este experimento, a adição de gema de ovo ao diluidor (Ringer + DMSO) teve efeito negativo sobre a motilidade e velocidade curvilinear do sêmen congelado de tambaqui, parâmetros importantes para a fertilização.

\section{Agradecimentos}

À Universidade Estadual do Ceará (UECE), ao Conselho Nacional de Desenvolvimento Científico e Tecnológico (CNPq), à Financiadora de Estudos e Projetos (FINEP) e ao Departamento Nacional de Obras Contra as Secas (DNOCS) pelo apoio no desenvolvimento da pesquisa.

\section{Referências}

1. Vieira MJAF, Carvalho MAM, Salmito-Vanderley CSB, Salgueiro CCM, Viveiros ATM, Moura AAAN, Nunes JF. Características do sêmen de tambaqui (Colossoma macropomum) em latitude equatorial. Archivos de Zootecnia. 2011;60(232):1263-1270. Disponível em: http://dx.doi.org/10.4321/S0004-05922011000400041

2. Maria AN, Azevedo HC, Santos JP, Silva CA, Carneiro CF. Semen characterization and sperm structure of the Amazon tambaqui Colossoma macropomum. J Appl Ichthyol. 2010;26(5):779-783. Disponível em: http:// dx.doi.org/ 10.1111/j.1439-0426.2010.01542.x

3. BRASIL. Boletim estatístico da pesca e aquicultura -2011. Brasília: Ministério da Pesca e Aquicultura; 2011. 60p. Portuguese. Disponível em: http:/www.mpa.gov.br/images/Docs/Informacoes e Estatisticas/ Boletim\%20MPA\%202011FINAL.pdf

4. Dairiki JK, Silva TBA. Revisão de Literatura: Exigências Nutricionais do Tambaqui - Compilação de Trabalhos, Formulação de Ração Adequada e Desafios Futuros. Manaus: EMBRAPA; 2011. 48p. Portuguese. Disponível em: https://www.infoteca.cnptia.embrapa.br/bitstream/doc/931300/1/Doc91.pdf

5. Carolsfeld J, Godinho HP, Zaniboni Filho E, Harvey BJ. Cryopreservation of sperm in Brazilian migratory fish conservation. J Fish Biol. 2003;63(2):472-489. Disponível em: http://dx.doi.org/10.1046/j.1095$\underline{8649.2003 .00170 . x}$

6. Carneiro PCF, Azevedo HC, Santos JP, Maria AN. Cryopreservation of tambaqui (Colossoma macropomum) semen: extenders, cryoprotectants, dilution ratios and freezing methods. Cryo Lett. 2012;33(5):385-393. Disponível em: http://www.ingentaconnect.com/content/cryo/cryo/2012/00000033/00000005/art00006

7. Varela AS Jr, Corcini CD, Streit DP Jr, Rizzoto G, Jardim RD, Lucia T Jr, Figueiredo MRC. Efeito crioprotetor de diferentes concentrações do dimetilsulfóxido no congelamento de sêmen de tambaqui Colossoma macropomum. Atlântica (Rio Grande). 2012;34(2):129-137. Disponível em: http://dx.doi.org/10.5088/ atlântica.v34i2.3175

8. Salmito-Vanderley CSB, Vieira MJAF, Leite LV, Oliveira FCE, Linhares FRA, Salgueiro CCM, Nunes JF. Meios de congelação para conservação de sêmen de peixes da família Characidae. Ciênc Anim. 2012;22(1):255268. Disponível em: http://www.uece.br/cienciaanimal/dmdocuments/CONERA_PALESTRA\%20 
\%2820\%29.pdf

9. Leite LV, Oliveira FCE, Nunes LT, Nunes JF, Salmito-Vanderley CSB. Criopreservação de sêmen de tambaqui com ACP ${ }^{\circledR}$ adicionado de gema de ovo. REPESCA. 2011;6(2):23-29. Disponível em: http://ppg. revistas.uema.br/index.php/REPESCA/article/view/421/635

10. Mansour N, Richardson GF, McNiven MA. Effect of extender composition and freezing rate on post-thaw motility and fertility of Arctic char, Salvelinus alpinus (L.), spermatozoa. Aquacult Res. 2006;37(9):862-868. Disponível em: http://dx.doi.org/10.1111/j.1365-2109.2006.01503.x

11. Kamaruding NA, Embong WKW, Abdullan RB. Frozen-thawed Sperm Motility Characteristics of African Catfish (Clarias gariepinus) by Using Glycerol or DMSO Based Extender. Int J Environ Sci Devel. 2012;3(1):49-55. Disponível em: http://repository.um.edu.my/28530/1/catfish.pdf

12. Muchlisin ZA, Hashim R, Chong ASC. Preliminary study on the cryopreservation of tropical bagrid catfish (Mystus nemurus) spermatozoa; the effect of extender and cryoprotectant on the motility after short-term storage. Theriogenology. 2004;62:25-34. Disponível em: http://dx.doi.org/10.1016/j.theriogenology.2003.05.006

13. Rana K, McAndrew B. The viability of cryopreserved tilapia spermatozoa. Aquaculture. 1989;76:335-345. Disponível em: http://dx.doi.org/10.1016/0044-8486(89)90085-9

14. Godinho HP, Viveiros ATM. Current status of sperm cryopreservation of Brazilian characiform fishes. In: Tiersch TR, Green CC. Cryopreservation in aquatic species. 2nd ed. Baton Rouge: The World Aquaculture Society; 2011. p. 875-884. Disponível em: https://www.academia.edu/3137728/Current_Status of Sperm Cryopreservation of Brazilian_Characiform Fishes

15. Streit DP Jr, Benites C, Moraes GV, Ribeiro RP, Sakaguti ES, Caldieri RF. Sêmen de pacu (Piaractus mesopotamicus) criopreservado com diluentes utilizados para sêmen de suínos. Cienc Anim Bras. 2006;7(3):289297. Disponível em: http://revistas.ufg.emnuvens.com.br/vet/article/view/409/384

16. Ninhaus-Silveira A, Foresti F, Veríssimo-Silveira R, Senhorini JA. Seminal analysis, cryogenic preservation, and fertility in matrinxã fish, Brycon cephalus (Günther, 1869). Braz Arch Biol Techn. 2006;49(4):651-659. Disponível em: http://dx.doi.org/10.1590/S1516-89132006000500015

17. Viveiros ATM, Oliveira AV, Maria NA, Orfão LH, Souza JC. Sensibilidade dos espermatozóides de dourado (Salminus brasiliensis) a diferentes meios de congelamento. Arq Bras Med Vet Zoot. 2009;61(4):883-889. Disponível em: http://www.scielo.br/pdf/abmvz/v61n4/v61n4a16.pdf

18. Ramirez-Merlano JA, Velasco-Santamaría YM, Medina-Robles VM, Cruz-Casallas PE. Cryopreservation effects on the sperm quality of cachama blanca Piaractus brachypomus (Cuvier 1818). Aquacult Res. 2011;42(6):738-745. Disponível em: http://dx.doi.org/10.1111/j.1365-2109.2011.02835.x

19. Cruz-Casallas PE, Medina-Robles VM, Velasco-Santamaría YM. Evaluación de diferentes crioprotectores para la crioconservación de espermatozoides de yamú (Brycon amazonicus). Rev Coml Cienc Pec. 2006;19(2):152-159. Disponível em: http://www.scielo.org.co/pdf/rccp/v19n2/v19n2a06.pdf

20. Maria AN, Viveiros ATM, Freitas RTF, Oliveira AV. Extenders and cryoprotectants for cooling and freezing of piracanjuba (Brycon orbignyanus) semen, an endangered Brazilian teleost fish. Aquaculture. 2006;260:298306. Disponível em: http://dx.doi.org/10.1016/j.aquaculture.2006.06.011

21. Fresneda A, Lenis G, Agudelo E, Olivera-Ángel M. Espermiación inducida y crioconservación de semen de cachama blanca (Piaractus brachypomus). Rev Coml Cienc Pec. 2004;17(4):46-52. Disponível em: http:// rccp.udea.edu.co/index.php/ojs/article/viewFile/185/182

22. Viveiros ATM, Godinho HP. Sperm quality and cryopreservation of Brazilian freshwater fish species: a review. Fish Physiol Biochem. 2009;35(1):137-150. Disponível em: http://dx.doi.org/10.1007/s10695-008$\underline{9240-3}$

23. Farias JO, Nunes JF, Carvalho MAM, Salgueiro CCM. Avaliação "in vitro" e "in vivo" do sêmen de 
Tambaqui (Colossoma macropomum) conservado a temperatura ambiente e criopreservado em água de coco. Rev Cient Prod Animal. 1999;1(1):44-58. Disponível em: http://www.ojs.ufpi.br/index.php/rcpa/article/ view/10/7

24. Menezes JTB, Queiroz LJ, Doria CRC, Menezes JB Jr. Avaliação espermática pós-descongelamento em tambaqui, Colossoma macropomum (Cuvier, 1818). Acta Amazon. 2008;38(2):365-368. Disponível em: http:// dx.doi.org/10.1590/S0044-59672008000200023

25. Viveiros ATM, Fessehaye Y, Ter Veld M, Schulz RW, Komen J. Hand-stripping of semen and quality after maturational hormone treatments, in African catfish Clarias gariepinus. Aquaculture. 2002;213:373-386. Disponível em: http://dx.doi.org/10.1016/S0044-8486(02)00036-4

26. Millinger J. Sperm et spermatozoïdes. In: Sexualité et reproduction des poissons. Paris : CNRS Éditions; 2002. p. 209-230. French.

27. Cosson J, Groison AL, Suquet M, Fauvel C, Dreanno C, Billard R. Studying sperm motility in marine fish: an overview on the state of the art. J Appl Ichthyol. 2008;24(4):460-486. Disponível em: http://dx.doi. org/10.1111/j.1439-0426.2008.01151.x

28. Alavi SMH, Cosson J. Sperm motility in fishes. (II) Effects of ions and osmolality: A review. Cell Biol Int. 2006;30(1):1-14. Disponível em: http://dx.doi.org/10.1016/j.cellbi.2005.06.004

29. Atencio VJ, Perez EJ, Espinosa JA, Pardo SC. Evaluación de dimetilacetamida como crioprotector para la crioconservación de semen de bocachico Prochilodus magdalenae. Arch Med Vet. 2013;45(2):151-158. Disponível em: http://dx.doi.org/10.4067/S0301-732X2013000200006

30. Streit DP Jr, Oliveira AC, Ribeiro RP, Sirol RN, Moraes GV, Galo JM, Digmayer M. Motilidade, vigor e patologias seminal in natura e pós criopreservação de Piaractus mesopotamicus. Bol Inst Pesca. 2009;35(2):159167. Disponível em: ftp://ftp.sp.gov.br/ftppesca/35 2_ 159-167.pdf

31. Linhares FRA, Salmito-Vanderley CSB, Carvalho MAM, Pinheiro RRR, Oliveira FCE, Nunes JF. Cinética e morfologia de espermatozoides de carpa comum criopreservados em água de coco em pó ACP-104. Arq Bras Med Vet Zootec. 2015;67(5):1313-1320. Disponível em: http://dx.doi.org/10.1590/1678-4162-8047

32. Solis-Murgas LD, Felizardo VO, Ferreira MR, Andrade ES, Veras GC. Importância da avaliação dos parâmetros reprodutivos em peixes nativos. Rev Bras Reprod Anim. 2011;35(2):186-191. Disponível em: http://www.cbra.org.br/pages/publicacoes/rbra/v35n2/RB353\%20Murgas\%20pag186-191.pdf

33. Melo-Maciel MAP, Salmito-Vanderley CSB, Leite LV, Oliveira FCE, Oliveira MS, Lopes JT, Nunes JF. Métodos de avaliação da qualidade do sêmen criopreservado de Characiformes brasileiros. Ciênc Anim. 2012;22(1):269-283. Disponível em: http://www.uece.br/cienciaanimal/dmdocuments/CONERA PALESTRA\%20\%2821\%29.pdf

34. Viveiros ATM, Nascimento AF, Orfão LH, Isaú ZA. Motility and fertility of the subtropical freshwater fish streaked prochilod (Prochilodus lineatus) sperm cryopreserved in powdered coconut water. Theriogenology. 2010;74(4):551-556. Disponível em: http://dx.doi.org/ 10.1016/j.theriogenology.2010.03.018

35. Nascimento AF, Maria AN, Pessoa NO, Carvalho MAM, Viveiros ATM. Out-of-season sperm cryopreserved in different media of the Amazonian freshwater fish pirapitinga (Piaractus brachypomus). Anim Reprod Sci. 2010;118:324-329. Disponível em: http://dx.doi.org/10.1016/j.anireprosci.2009.07.002

36. Verstegen J, Iguer-Ouada M, Onclin K. Computer assisted semen analyzers in andrology research and veterinary practice. Theriogenology. 2002;57(1):149-179. Disponível em: http://dx.doi.org/10.1016/S0093$\underline{691 X(01) 00664-1}$ 